\title{
Pharmacogenetics in Psychiatry Satellite Meeting at the American College of Neuropsychopharmacology, 2000
}

The Pharmacogenetics in Psychiatry satellite meeting of the American College of Neuropsychopharmacology met on December 13, 2000 in San Juan, Puerto Rico to discuss new developments in genomics that relate to pharmacogenetic studies as well as to continue previous meeting's discussions on appropriate guidelines for pharmacogenetic studies. In contrast to previous years, in which the majority of discussion was related to clinical trials issues as they pertain to pharmacogenetics, this year's meeting was highlighted by increased discussion about methodological advances in genomic information, genotyping technology, and statistical methodologies. The agenda for the meeting included four areas of discussion: the feasibility of whole genome association studies, dealing with gene-gene and gene-environment interactions, the role of academia versus private industry in pharmacogenetics, and the theoretical and practical issues in collecting pharmacogenetic study groups sufficiently large to detect relatively small gene effects.

\section{STATUS OF WHOLE GENOME ASSOCIATION STUDIES}

Several recent developments have suggested that genome scans for pharmacogenetic traits may be possible in the near future. Most importantly, a working draft of the human genome sequence has been completed during the past year and preliminary data suggest that the total number of human genes may be as low as 30-35,000. As studies that have comprehensively scanned multiple genes for allelic variation report an average of 1-2 non-synonymous (result in an amino acid substitution) common coding region single nucleotide polymorphisms (cSNPs) per gene (Cargill et al. 1999), these data suggest that there may be less than 75,000 common cSNPs in the human genome. Celera Genomics recently reported the identification of over 2 million SNPs in their data base and the SNP consortium, a industry sponsored group of academic and industrial sites, has identified over 1,000,000 SNPs and made them publicly available. Therefore, cSNPs may soon be identified in many, if not all human genes. Finally, large scale clinical trials are underway in academia (for example the NIMH-funded CATIE trial in schizophrenia), as well as in the pharmaceutical industry, that should provide robust samples for pharmacogenetic studies. Therefore, it may be feasible to consider "whole genome cSNP association" studies, in which the common cSNPs throughout the genome (or perhaps restricted to those expressed in the CNS) would be genotyped in large scale clinical trial populations (of at least 1000 patients) in order to identify the associations between individual SNPs and drug response measures such as efficacy and adverse side effects. The advantages of this approach are that almost every gene would receive some genotyping coverage, and that the cSNPs would have the highest a priori probability of affecting function, although promoter and regulatory region polymorphisms should also be considered good candidate polymorphisms.

Several concerns were raised with this proposal and discussed by the group. First, testing of 75,000 SNPs would raise significant statistical problems, such as correction for multiple testing, that would markedly reduce the study power. However, an advantage of the case control association methodology is its enhanced power in comparison to family based association methods or traditional linkage approaches. For example, Risch and Teng (1998) examined the relative power of the case control design versus family based approaches under a number of different genetic models. Under 
most genetic models, the case control design was markedly more efficient than family based designs, with sample sizes of less than 1000 usually sufficient to detect genes of relatively modest effect, even with extremely conservative significance levels $\left(\alpha=5 \times 10^{-8}\right)$ and with minor allele frequencies ranging from $5 \%$ to $70 \%$. Therefore, even if one treated each cSNP as completely independent of each other, Bonferroni correction accounting for genotyping of 75,000 SNPs would still result in sufficient power to detect several genes of modest effect. Clearly, some genes of small effect would be missed with this conservative threshold; however, the identification of even a single gene that influences drug response would be of significant merit because it could implicate specific pathways in drug efficacy, as well as render follow-up pharmacogenetic studies more powerful by providing data on the effects of at least one gene on phenotypic variation.

Another concern with the case-control association design is the potential for ethnic stratification between subject groups. In the case of psychiatric pharmacogenetic studies, this might arise in a study comparing candidate allele frequencies between drug responders versus nonresponders (Malhotra and Goldman 1999). If the candidate allele frequency varies between ethnic groups, and responders and nonresponders are not ethnically matched, a significant difference in allele frequencies could be detected that is not associated with drug response. Unfortunately, ethnic variation in allele frequencies is not uncommon. For example, Chang and colleagues (1996) have found that frequencies for the 4-repeat allele of the D4DR 16 amino acid repeat varies between 16 and $96 \%$ between populations. The D2DR Taq I A1 allele is twice as frequent in African Americans and four times as frequent in some American Indians as compared with Caucasian Americans (Goldman et al. 1993). Fortunately, "genomic control" methods are now under development to help account for ethnic stratification in association studies. Genomic control techniques are based upon the idea that study groups (cases vs. controls, responders vs. nonresponders etc...) can be assessed for the presence of stratification by assessing the allele frequency of markers, unlinked to the phenotype of interest, in each group. Pritchard and Rosenberg (1999) determined that no more than 40 unlinked markers, and perhaps less, are necessary to achieve $95 \%$ probability of detecting stratification in study groups of over 200 subjects. If stratification is detected, subjects can be removed until stratification is not present, or correction factors can be introduced that account for the level of stratification between groups. Both approaches reduce study power but minimize the risk that the case-control design results in a false positive or negative result. In the case of an association study involving $75,000 \mathrm{cSNPs}$, it would be relatively easy to include a sufficient number of markers known to be unlinked to drug response phenotypes, and therefore assess the potential for stratification empirically.

Finally, genotyping costs were discussed as a potential limitation of whole genome association studies. With genotyping costs as high as $\$ 1$ per SNP locus, the cost of genotyping 75,000 SNPs in 1000 patients would be prohibitive (\$75 million). The case-control methodology with unrelated patients, however, allows strategies that could significantly reduce genotyping expenditures, for example, by pooling hundreds of DNA samples for "screening genotyping" procedures prior to beginning more intensive individual genotyping. Moreover, the costs of SNP genotyping continues to decrease with some estimates of costs as low as \$.03-.05 per SNP genotype in the near future. At these costs, the total cost for genotyping 75,000 cSNPs in 1000 patients would be reduced to \$2-4 million-a not inconsiderable sum, but well within the purview of many biotechnology and pharmaceutical firms with interests in pharmacogenetics.

Several other issues must be addressed prior to conducting a whole genome cSNP association study, including ethical issues of privacy and utilization of data by non-academic groups such as private industry or insurance companies. Nevertheless, the group concluded that, with the pace of recent technological and statistical developments, the potential for such studies merits careful consideration.

\section{GENE-GENE AND GENE- ENVIRONMENT INTERACTIONS}

The complexity of pharmacogenetic traits have suggested that analyses of multiple genes may be required to more fully assess drug response. Initial studies of schizophrenia patient's response to clozapine focused on single genes with the hope that a single gene might account for a large proportion of the phenotypic variance. With the cloning of the dopamine D4 receptor gene (DRD4), it was hoped, because of the relatively high affinity of D4 for clozapine, that genetic variation in DRD4 would predict clinical response (Shaikh et al. 1995). Although there is still a remote possibility that $D R D 4$ is a major determinant of clozapine response, the negative pharmacogenetic results with the major polymorphisms in DRD4 render it unlikely that DRD4 will contribute in a major way to prediction of clozapine response.

Similarly, studies of other single serotonin and dopamine receptor subtypes have not resulted in a strong positive association with clozapine response as yet (Masellis et al. 2000), with the possible exception of 5-HT2A receptor genetic variation (Arranz et al. 1998). Given the relative lack of success using single candidate genes in prediction of clozapine response, recently research- 
ers have begun to investigate several genes simultaneously. The most ambitious attempt to date has been by Arranz et al. (2000), who examined more than one dozen candidate genes including histamine receptors and adrenergic receptor genes in an exploratory fashion. A combination of six polymorphisms resulted in $76.7 \%$ success in the prediction of clozapine response and a sensitivity of $95 \%$. Since this result is exploratory, it will be important to replicate this analysis in independent data sets of well-characterized patients.

An alternative approach in psychiatric pharmacogenetics is to select a sub phenotype of schizophrenia for more limited but hopefully more precise analysis. One such phenotype is the side effect of tardive dyskinesia (TD) seen in up to $30 \%$ of patients treated with traditional neuroleptic medications. The phenotype of TD has the advantage of being objectively visible and is relatively amenable to scaled scoring in terms of the degree of severity. In contrast to genetic studies of clozapine response, the genetic studies of TD have yielded quite promising and replicable results.

Several groups have independently shown that the dopamine D3 receptor Ser9Gly polymorphism is associated with risk for TD (Steen et al. 1997; Basile et al. 2000; Segman et al. 1999; Macciardi et al., unpublished data). Each group found that the glycine allele conferred significantly elevated risk for TD. Basile et al. (2000) showed further that the glycine allele occurred at a higher rate in African Americans, and was associated with TD (as measured by the Abnormal Involuntary Movement Scale) in both Caucasian and African American patients. A single study in a German population of patients with schizophrenia did not find an association between the D3 gene and TD. (Rietschel et al. 2000). Under the assumption of the involvement of multiple genes, Basile and colleagues then examined the role of the cytochrome P450 enzyme genes CYP2D6 and CYP1A2. The CYP2D6 gene variation did not predict risk for TD, however, the CYP1A2 polymorphism did show a significant association.

In view of the two genes, dopamine $\mathrm{D} 3$ receptor and CYP1A2, each contributing to the risk for TD, a genegene interaction analysis has begun with the data set from the Toronto group. First the various models of gene-gene interaction have been delineated in matrices representing combinations of dominant, recessive, additive and epistatic effects. The D3 and CYP1A2 genetic data were then fitted to each of these models. Thus far in the analyses, the best fit is an additive co-dominant model of D3 and CYP1A2 interaction (Basile, Kennedy and Macciardi, unpublished data). Furthermore, the environmental factor of smoking was incorporated into the genetic analysis and this improved the prediction power (Basile et al. 2000). The overall model now accounts for more than $55 \%$ of the variance in risk for TD. This investigation of gene-gene and environment inter- action represents an initial step toward a multigene model that may account for most of the variance in risk for TD.

\section{THE ROLE OF ACADEMIC RESEARCHERS VERSUS PRIVATE INDUSTRY IN PHARMACOGENETICS}

A third area of discussion was the relationship between academic researchers and private industry in pharmacogenetics. The current intense interest from pharmaceutical and biotechnology companies in the emerging human genome sequence has resulted in substantial increases in financial support dedicated to research into human biology. Pharmacogenetics is a particular focus of activity because of the potential for the development of genetic tests for clinical response and adverse side effects. These in themselves will have commercial value as licensed clinical tests. Pharmacogenetics also has the potential to identify the true therapeutic targets of antipsychotics, through the correlation of genetic variation in a given receptor with clinical response. Pharmacogenetics information may also be used to develop niche markets for drugs, which may not be useful in the whole disease population but have benefit for a genetically-defined subgroup. All this information will assist in the development of future drugs. Improving the safety of drugs by identifying and protecting those patients vulnerable to side effects may also broaden the indications for drugs with a known side effect risk; for example improving the safety of clozapine through a test for agranulocytosis may broaden its use in psychosis to non-treatment-resistant forms.

A common problem facing academic researchers, not least those involved in pharmacogenetics, is funding. Even for a well-funded area of science such as genetics, obtaining funding for pharmacogenetics research has been difficult. This is partly because it is an emerging area whose general utility has not been fully appreciated. Increasing sums are being spent on pharmacogenetics in industry, however. Until recently, pharmaceutical companies have adopted research strategies mainly based on 'add-on' genetic studies allied to clinical trials of drugs. However, clinical trials are often small and use selected patient groups from multiple geographic origins, introducing confounding variables for genetic studies.

The most beneficial approach for pharmacogenetics may be cooperation between academic groups and industry. Good quality prospective pharmacogenetic experiments require hundreds of subjects and are consequently expensive to set up and run. In addition, the cost of genotyping large numbers of SNPs and other polymorphisms, for example, when considering a genome-wide scan for pharmacogenetic associations, is 
prohibitive for academic groups. Furthermore, alongside their academic counterparts, companies are developing exciting new computational approaches such as human haplotype analysis, which will benefit academic researchers.

Thus partnerships are emerging where pharmaceuti$\mathrm{cal} /$ biotechnology companies will fund clinical and laboratory staff to collect pharmacogenetic samples, and the company will also provide bioinformatic, statistical and genomic/genotyping expertise and resources. The academic researcher is able to provide access to research volunteers, clinical expertise and intellectual input into the partnership. The most likely research scenario is a sample collected for exclusive shared use by the company and the academic researcher over a set time period (say five years), with a share of intellectual property rights for the academic investigators/University (perhaps of a few percent) in addition to research funding for the university and joint access to the sample collection for co-ordinated commercial and academic projects.

The group considered the appropriate methods to enhance such joint ventures. To be avoided is the concept of 'sale' of samples, which is becoming increasingly controversial in many countries, especially the U.K. The scenario where academic/clinical researchers act solely to collect research volunteers for confidential industry research should be viewed with caution because of the need to maintain intellectual input and openness. A good partnership will include reasonable guidelines for publication of data in peer reviewed journals without excessive delay. This is an essential component of academic involvement. Any delay in publication should be agreed to in advance, and compromise should be sought between commercial considerations and dissemination and scrutiny by the international community. Robust ethical review is required for this approach. It is necessary to ensure that the research subjects are fully informed that the research is commercial in nature and that they will not receive a share of the profits, and that their rights (for example with respect to immortalised cell lines) are fully respected. The best way forward for commercial pharmacogenetic research should be an equitable and co-operative partnership between academia and industry.

\section{STUDY DESIGN AND SAMPLE SIZE CONSIDERATIONS}

The group also considered issues surrounding the design and sample size of pharmacogenetic studies of antipsychotic drugs. As genetic and non-genetic influences presumably interact to determine antipsychotic drug response, it is likely that a single genetic variant will only have a very limited effect on a specific out- come measure. Sample size considerations in order to detect such a variant are based on theoretical assumptions as empirical data are lacking. Theoretical statistical calculations show that depending on the frequency of the disease allele in the general population, from 350 to 5800 patients and controls may be required for identification of a susceptibility gene, given a genotypic risk ratio of 2 . The smaller the genotypic risk ratio becomes, the larger sample sizes are needed; for a genotypic risk ratio of 1.5 the required sample sizes range from 950 to 19,500 (Risch and Merikangas 1996). Novel molecular genetic technologies and advanced biometric methods are available to conduct marker-intensive searches for association with the given response/side-effect. Therefore, the availability of large patient samples with standardized phenotype characterization is an important prerequisite for the identification of genes influencing patients' response to medication. As the collection of these samples currently constitutes a major limiting component, the group considered the merits of multicenter studies. Pursuing this approach, it has to be kept in mind that genetic heterogeneity between samples, differences in diagnostic approaches, or sampling effects, may blur the limited effect of single variants. In order to overcome these problems, we initiated in 1998 a consensus conference which brought together researchers in the field to discuss the possibilities for standardization of assessment procedures (Rietschel et al. 1999). The application of these tools has now led to an international (Israel, Germany, Austria, Canada, US, Sweden) multi-center study of the influence of the dopamine D3 receptor gene on the development of tardive dyskinesia (TD) in 795 patients exposed to long-term neuroleptic treatment. Despite the variability among groups, the results are significant and support an association of the dopamine D3 receptor gene with TD. The large sample size also provides an opportunity to examine the relationship of genetic polymorphism to variables that could not be explored in individual samples. Hopefully, this study will represent a first step in the development of large scale international collaborations in the field of psychiatric pharmacogenetics.

\section{CONCLUSION}

The meeting concluded with the assessment that further meetings on this topic will be worthwhile to assess the progress of this rapidly developing field. Therefore, an annual Pharmacogenetics in Psychiatry Meeting has been organized with the first meeting to take place in New York City on April 12-13, 2002. Investigators interested in attending should contact Anil K. Malhotra at malhotra@lij.edu for program and registration information. 
Anil K. Malhotra, M.D. Hillside Hospital, Unit of Molecular Psychiatry Glen Oaks, NY

James L. Kennedy, M.D. Centre for Addiction and Mental Health Section of Neurogenetics Ontario, Canada

David A. Collier, Ph.D. The Institute of Psychiatry Division of Psychology Medicine

Denmark Hill, London

Marcella Rietschel, M.D. University of Bonn

Department of Psychiatry Bonn, Germany

\section{REFERENCES}

Arranz MJ, Munro J, Birkett J, Bolonna A, Mancama D, Sodhi M, Lesch KP, Meyer JF, Sham P, Collier DA, Murray RM, Kerwin RW (2000): Pharmacogenetic prediction of clozapine response. Lancet 355:1615-1616

Arranz MJ, Munro K, Birkett J, Kirov G, Murray RM, Collier DA, Kerwin RW (1998): Meta-analysis of studies on genetic variation in 5-HTA receptors and clozapine response. Schizophr Res 32:93-99

Basile VS, Ozdemir V, Masellis M, Walker ML, Meltzer HY, Lieberman JA, Potkin SG, Alva G, Kalow W, Macciardi FM, Kennedy JL (2000): A functional polymorphism of the cytochrome P450 1A2 (CYP1A2) gene: association with tardive dyskinesia in schizophrenia. Mol Psychiatry 5:410-417

Cargill M, Altshuler D, Ireland J, Sklar P, Ardlie K, Patil N, Lane C, Lim E, Kalyanaraman N, Nemesh J, Ziaugra L, Friedland L, Rolfe A, Warrington J, Lipshutz R, Daley GQ, Lander ES (1999): Characterization of single-nucleotide polymorphisms in coding regions of human genes. Nat Genet 22:231-238

Chang FM, Kidd JR, Livak KJ, Pakstis AJ, Kidd KK (1996): The world-wide distribution of allele frequencies at the human dopamine D4 receptor locus. Hum Genet 98:91-101
Goldman D, Brown GL, Albaugh B, Robin R, Goodson S, Trunzo M, Akhtar L, Lucas-Derse S, Long J, Linnoila M (1993): DRD2 dopamine receptor genotype, linkage disequilibrium and alcoholism in American Indians and other populations. Alcoholism Clinical and Experimental Research 17:199-204

Malhotra AK, Goldman D (1999): Benefits and pitfalls encountered in psychiatric genetic association studies. Biol Psychiatry 45:544-550

Masellis M, Basile VS, Ozdemir V, Meltzer HY, Macciardi FM, Kennedy JL (2000): Pharmacogenetics of antipsychotic treatment: lessons learned from clozapine. Biol Psychiatry 47:252-266

Pritchard JK, Rosenberg NA (1999): Use of unlinked genetic markers to detect population stratification in association studies. Am J Hum Genet 65:220-228

Rietschel M, Kennedy JL, Macciardi F, Meltzer HY (1999): Application of pharmacogenetics to psychotic disorders: the first consensus conference. Schizophr Res 37:191-196

Rietschel M, Krauss H, Muller DJ, Schulze TG, Knapp M, Marwinski K, Marold AO, Paus S, Grunhage F, Propping P, Maier W, Held T, Nothen MM (2000): Dopamine D3 receptor variant and tardive dyskinesia. Eur Arch Psychiatry Clin Neurosci 250:31-35

Risch N, Merikangas K (1996): The future of genetic studies of complex human diseases. Science 273:1516-1517

Risch N, Teng J (1998): The relative power of family-based and case-control designs for linkage disequilibrium studies of complex human diseases I. DNA pooling. Genome Research 8:1273-1288

Segman R, Neeman T, Heresco-Levy U, Finkel B, Karagichev L, Schlafman M, Dorevitch A, Yakir A, Lerner A, Shelevoy A, Lerer B (1999): Genotypic association between the dopamine D3 receptor and tardive dyskinesia chronic schizophrenia. Mol Psychiatry 4:247-253

Shaikh S, Collier DA, Sham P, Pilowsky L, Sharma T, Lin LK, Crocq MA, Gill M, Kerwin R (1995): Analysis of clozapine response and polymorphisms of the dopamine D4 receptor gene (DRD4) in schizophrenic patients. Am J Med Genet 60:541-550

Steen VM, Lovlie R, MacEwan T, McCreadie RG (1997): Dopamine D3-receptor gene variant and susceptibility to tardive dyskinesia in schizophrenic patients. Mol Psychiatry 2:139-145 